\title{
Correspondence
}

\section{Bain circuit delivery tube obstructions}

To the Editor:

Bain circuits which we presently use are designed with a coloured delivery tube so that kinks or obstructions to the tube will be easily seen. We received a new Bain circuit which appeared intact, but when it was placed on the anaesthetic machine, it provided no gas flow at the patient end. On examining the circuit, the delivery tube was obstructed and kinked at the proximal end. The obstruction was not visible, as it was inside the opaque connecter at the proximal end of the circuit. This is clearly seen in the radiograph (Figure).

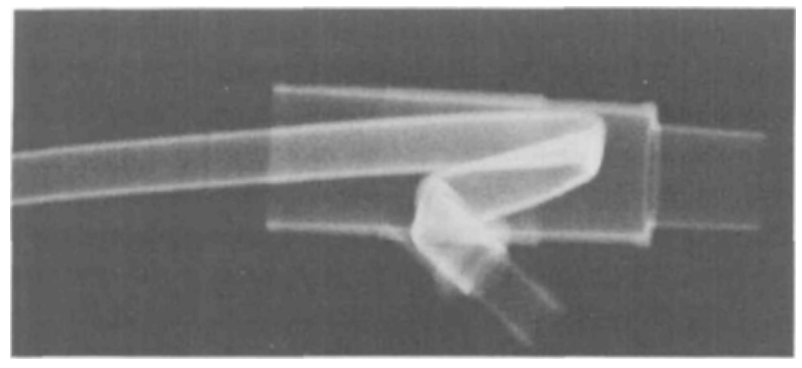

FIGURE Inflow obstruction of a Bain circuit fresh gas flow delivery tube.

This matter is drawn to your attention firstly to caution anaesthetists to use appropriate tests to ensure the integrity of the Bain Circuit prior to commencing an anaesthetic and, secondly, in the hope that manufacturers of couxial circuits would take this report as a challenge to provide circuits which allow complete visibility of the gas delivery tube over the entire length of the circuit from the common gas outlet to the patient end.

Gerald V. Goresky

Director

Department of Anaesthesia

Alberta Children's Hospital

\section{Continuous subcutaneous injection of ketamine for cancer pain}

Subanaesthetic doses of ketamine have been administered as an analgesic to injured, postoperative, and cancer patients by intravenous, ${ }^{1}$ intramuscular, ${ }^{2}$ epidural, ${ }^{3}$ and intrathecal ${ }^{4}$ routes. We report the effectiveness of ketamine administered via another route, continuous subcutaneous injection of ketamine which was effective to relieve cancer pain in 13 out of 18 patients. The advantages of the method of continuous subcutaneous injection have been previously described. ${ }^{5,6}$

All patients were in the terminal stage of cancer and had intractable pain. Analgesics, such as morphine and pentazocine, had became ineffective for pain control. Ten $\mathrm{mg}(0.2 \mathrm{ml})$ of ketamine hydrochloride was injected initially, which was followed by a continuous subcutaneous injection, at a rate of $10 \mathrm{mg} \cdot \mathrm{hr}^{-1}$, using a batterydriven infusion pump (Nipro SP-10, Nipro, Osaka). The rate of administration was titrated by increments of 2.5 $\mathrm{mg}$ daily up to $15 \mathrm{mg} \cdot \mathrm{hr}^{-1}$ to meet the patients' requirements. The maximum rate was arbitrarily fixed at 15 $\mathrm{mg} \cdot \mathrm{hr}^{-1}$ based on the reports of Idval et al. ${ }^{7}$ and Nimmo et al. ${ }^{8}$ since the distribution volume per body weight might be decreased in terminal patients and body weight might not be helpful in calculating doses. Pain was subjectively graded by patients into four stages: 0 (none); 1 (slight); 2 (obvious but tolerable); 3 (intolerable). Patients were requested to assess the maximum pain at three assessment periods of $0-8,8-16,16-24$ hours. Three consecutive scores of 0 and 1 were considered to be effective.

Very low doses of ketamine $\left(2.5-15 \mathrm{mg} \cdot \mathrm{hr}^{-1}\right)$ produced analgesia in the region of both the spinal and the cranial nerves with alert consciousness. It was not effective in three patients and was of questionable benefit in two. Ketamine was given as the sole analgesic in four patients for 10-48 d. Side-effects consisted of injection site inflammation (six), salivation (two), and insomnia (two). In two patients with long-term administration (202 and 147 days), ketamine's analgesic potency seemed to decrease. An acute tolerance to ketamine analgesia has been postulated, ${ }^{9}$ however, the time courses of the present cases did not support this. The decrease in effect was probably due to exaggeration of pain rather than the development of tolerance. 